\title{
Ergonomics and Musculoskeletal Health of the Surgeon
}

\author{
Andrew T. Schlussel, DO, FACS, FASCRS ${ }^{1}$ \\ ${ }^{1}$ Department of Surgery, Madigan Army Medical Center, Tacoma, \\ Washington \\ 2 Division of Colon and Rectal Surgery, University of Massachusetts \\ Memorial Medical Center, Worcester, Massachusetts
}

Justin A. Maykel, MD, FACS, FASCRS²

\begin{abstract}
Address for correspondence Justin A. Maykel, MD, FACS, FASCRS, Division of Colon and Rectal Surgery, University of Massachusetts Memorial Medical Center, 67 Belmont Street, Suite 201, Worcester, MA 01605 (e-mail: justin.maykel@umassmemorial.org).
\end{abstract}

Clin Colon Rectal Surg 2019;32:424-434.

\begin{abstract}
Keywords

- ergonomics

- occupational injury

- surgeon health

Surgeons face hazardous working conditions due to ergonomic deficiencies found throughout the operating room. More than $80 \%$ of surgeons have succumbed to a work-related injury or illness as a consequence of sustaining awkward or static positions throughout lengthy operations over a career. While the focus of the procedure is directed to the patient, there is little concurrent regard for proper posture or spinal stability. Even when symptoms of discomfort are experienced, they are often ignored. This results in decreased production and may ultimately threaten surgical careers. Surgeons are often unaware of recommendations or guidelines designed to improve their comfort while operating. Furthermore, there is a significant lack of any formal ergonomic education, especially for minimally invasive procedures. Several modifiable risk factors can be adjusted in the operating room to provide a safer working environment. In addition, strengthening, stability, and exercise programs directed by a trained therapist may be employed to improve the surgeon's musculoskeletal health.
\end{abstract}

\section{Case Example by Justin Maykel, MD}

Earlier in my career, I never considered the impact surgery would have on my own musculoskeletal (MSK) health. As I progressed into my late thirties, I started to experience back and hip pain so severe that I could barely walk after long days in the operating room. I started wearing a back brace while operating. I began taking naproxen on a regular basis. I never told anyone out of fear of stigma. I wanted to keep building my practice and not slow down. At first, I assumed it would just get better. Eventually, as my symptoms progressed, I tried everything-stretching, braces, massage therapy, acupuncture, physical therapy, chiropractor, steroid injections.

A magnetic resonance imaging (MRI) revealed torn cartilage around my hip. This resulted from years of repetitive, positional trauma working in the operating room without regard for proper ergonomics. It required surgery and an associated 6-week recovery, during which I was sidelined out of work.
I was shocked to learn that nearly all of my colleagues had chronic MSK conditions related to the physical demands of our job. Surgeons are like professional athletes, who require conditioning to keep them performing at the top of their game. We need to improve our working environment and maintain our physical health to make sure that we can perform optimally and maintain career longevity. Step 1 is to recognize the reality of the situation. Step 2 is to do something about it, and therefore we welcome you to keep reading.

\section{Introduction}

At the conclusion of medical school, young physicians embark upon their professional journey with the acceptance of the Hippocratic Oath, affirming they will do no harm. As admirable as this pledge may be, surgeons rarely consider the personal harm that may ensue from years of operating in hazardous conditions. A recent report from the American College of Surgeons' Surgery News Bulletin has addressed the mounting health concerns of surgeons, declaring "This has to ISSN 1531-0043. 
change, for how can we provide excellent care to the patient if we cannot maintain our own health and safety." 1

Surgeons are conditioned and trained in a high-paced, productivity-driven environment, where the thoughts or complaints related to stress and fatigue are often discouraged or simply ignored. Medical students and residents are generally young, flexible, and limber. Their senior surgeons would be unlikely to admit or address any personal weakness or limitations to their trainees. This flawed relationship represents a critical barrier to open dialog and progress. The consequences are severe and relate to both physical and mental health. ${ }^{2}$

Despite all the advances in medical technology, operating rooms are still not designed ergonomically nor with the surgeon's health in mind. Operating tables have limited mobility, floor surfaces are hard, and monitors are positioned incorrectly. Furthermore, the complexity and duration of operations have evolved, often related to the epidemic of morbid obesity in our population. The surgeon stands and operates in awkward positions for multiple hours. As a consequence, reports have demonstrated that 50 to $85 \%$ of practicing surgeons worldwide experience regular MSK discomfort in the neck, shoulders, and back. Endoscopic procedures are another risk factor for the incidence of hand and finger injuries (42\%), as well as neck pain (11\%). These symptoms may all lead to chronic disorders over time. ${ }^{3-10}$

Those at a greater risk of injury include surgeons with a history of preexisting neuromuscular disorders such as carpal tunnel syndrome, tendonitis, and arthritis. ${ }^{11,12}$ In addition, case volume, duration, and disproportionate hand size to instrumentation are associated with a greater rate of neurapraxia. ${ }^{13-15}$ Maintaining a static position for an extended period of time, especially when operating in the extremes of quadrants of the abdominopelvic cavity, increases the physical workload endured by the MSK system. Davis et al have reported that more than $40 \%$ of surgeons experienced one or more injuries in the workplace. These injuries affect operative performance in half of these surgeons, whereas 20 to $30 \%$ missed work or reduced the number of operations performed as a direct result of their injury. Sadly, only $20 \%$ of surgeons officially reported their injury. ${ }^{16}$ As a result, the cost for medical treatment of occupational related injuries have exceeded 100 billion dollars annually, a significant economic burden on society. ${ }^{17,18}$

It is critical that our profession focuses not only on the patient but also on improving the physical and mental wellbeing of the provider. Surgeons are not invincible and should be encouraged to reveal their limitations and recognize the potential risks related to performing surgery. Failing to embrace these issues will affect a surgeon's ability to function, resulting in diminished career satisfaction, fatigue, and burnout. ${ }^{8,19}$ Unfortunately, many surgeons never receive a formal education or are even aware that guidelines exist to help prevent these prevalent occupational injuries. $9,20,21$ Even more disappointing, when properly trained and educated, only $60 \%$ of surgeons actually incorporate appropriate ergonomic designs in their surgical practices. ${ }^{21}$

\section{Human Factors and Ergonomics}

To master surgeon safety in the operating room, one must understand the science of ergonomics. The word ergonomics stems from the Greek words "ergon," meaning work, and "nomos," meaning natural laws or arrangements. It is the study of people at work and how the working environment is designed to suit the worker. ${ }^{22}$ Ergonomics relates to the interaction between person and machine, and the subsequent interaction the machine plays on the person, with the goal of optimizing how technology is used. ${ }^{11,23,24}$ Combined as a complete system, the principles of ergonomics use anatomy, physiology, psychology, and engineering to create a favorable environment that mitigates MSK complaints and optimizes performance and efficiency. ${ }^{24}$

The conditions that surgeons withstand have been compared with those of certain industrial workers. ${ }^{25}$ Unfortunately, little basic science research has been implemented to address and evaluate the ergonomics in the operating room. ${ }^{15,26}$ Integrating the field of ergonomics into medicine, especially surgery, can be challenging as multiple factors cannot be manipulated. These include the patient's body habitus or the necessity to remain within the constraints of a sterile surgical field.

The discipline of ergonomics has evolved significantly since its inception in the early 1900 s. $^{27,28}$ Research has been performed and employed in large industrial companies, the military, and sports training to maximize performance while reducing errors and injury. ${ }^{11}$ More recently, focus has been drawn to the medical environment. Methods to mobilize and care for patients in intensive care units, gastrointestinal endoscopy suites, and medical-surgical wards have been implemented to specifically minimize back injuries. ${ }^{29}$ As the incidence of cervicobrachial injuries rose in the dental profession, this encouraged the redesign of equipment and techniques to mitigate further health risks to the provider. ${ }^{30,31}$ Similar responses are more complex in the field of surgery. The wide variety of operative techniques, disease locations, and patient position all alter the operating room environment. These factors contribute to how the operative field is visualized, the way surgical instruments are manipulated, and, ultimately, surgeon stance and posture. ${ }^{11,26}$ These variables span open, laparoscopic, and robotic approaches.

\section{Body Positioning}

"Posture" is defined as the spatial arrangements of body parts as they align to perform a task. This is an interface between segmental joints of the body and muscles crossing these joints to protect the body's support structures and prevent damage in any position. These factors are influenced by the task, environment, working tools, and biologic characteristics of the worker. ${ }^{32}$ A healthy, stable posture occurs when one is standing upright without a rounded back. The spine should remain straight to maintain the natural lordotic and kyphotic curves of the vertebrae. The elbows should be facing outward, the shoulder blades brought down and inward, while tucking in the core and buttocks. Focus on a 


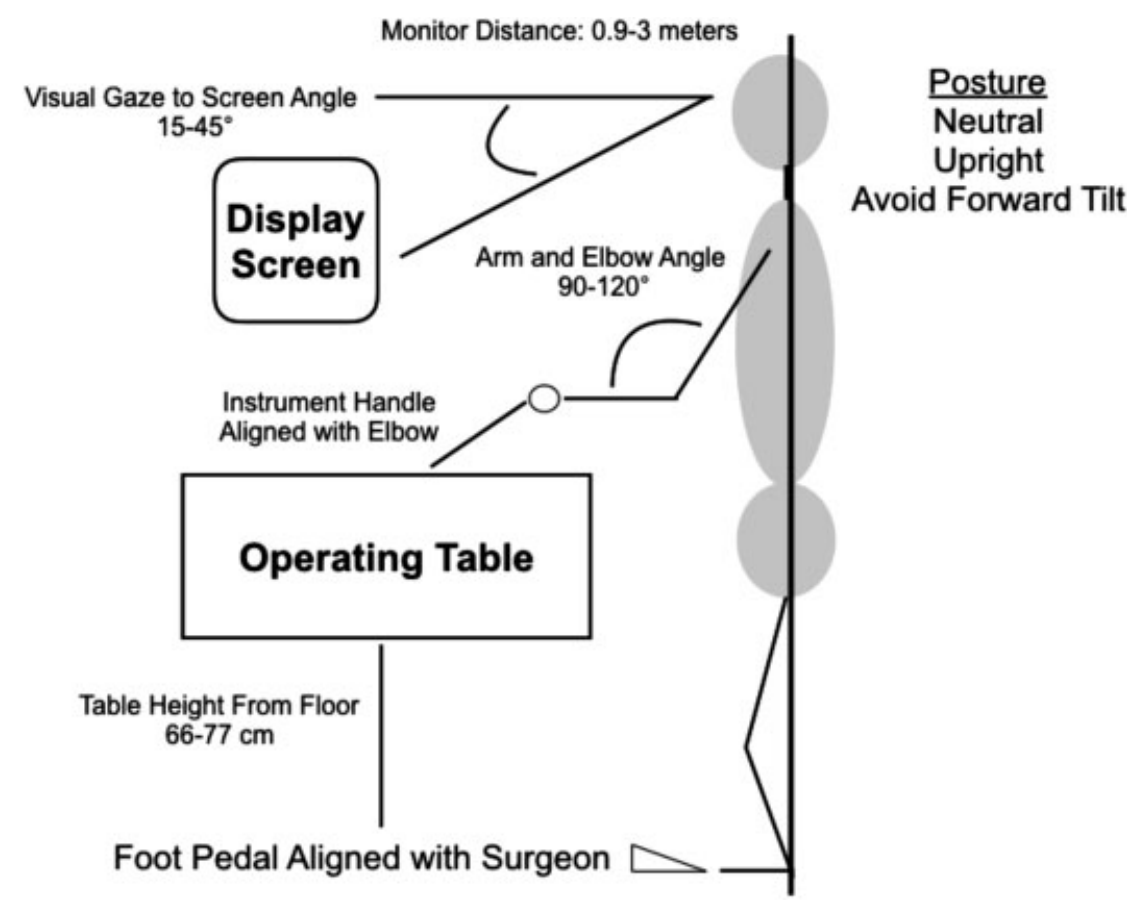

Fig. 1 Optimal ergonomic position.

line of gravity transecting all the spinal curves, and this will provide a stable structure and base of support ( $\mathbf{- F i g . 1}$ ). The best means of preventing work-related injuries are understanding and recognizing how a stable posture feels and understanding the downsides of a faulty posture that will lead to MSK injuries. ${ }^{33,34}$ This understanding is not innate and requires coaching and training to develop muscle memory.

One must not underestimate the physical exertion and mechanical load placed on the body while operating. An alteration in body mechanics when placed in constrained, asymmetric, and prolonged positions results in tissue stress and injury due to overexertion or imbalance. Improper working postures place muscles, tendons, and ligaments at risk. Abnormal loads and strains result in damage to bones, joints, and cartilage. Furthermore, neurapraxia may occur because of nerve compression or ischemia. ${ }^{35}$ In more than $50 \%$ of surgeons, posture alone has contributed to their symptoms. ${ }^{4}$

Rosenblatt et al described the three most common errors in ergonomic posture: excessive forward head position, sustained uncomfortable elevations, and asymmetry in weight-bearing. ${ }^{36}$ When the head is maintained in a too far forward posture, it results in tension and pain in the upper torso, ultimately causing degenerative changes in the cervical spine and shoulder girdle. For every inch that the head moves forward in space, the relative weight on the spine increases by 10 pounds, resulting in an increased demand on the joints supporting the head. Regrettably, many of these positions are performed subconsciously from poor habits that are adopted overtime. Failure to become aware of these positions increases the workload placed on the spinal column, and microtrauma accumulates in the tissue. ${ }^{37}$ In addition, the frequent uncoordinated and repetitive motions of the upper extremities and prolonged static head and back postures inflict unnecessary stress on the spinal column. ${ }^{38}$ During open surgery, a common posture employed by the surgeon is a head- and back-bent position, with a twisted torso. ${ }^{10}$ Laparoscopic operations require a head- and backstraight stance with an upright skeleton to allow the eyes to focus on the display screen, whereas the arms are commonly placed in a continuous abducted and internally rotated position, a position associated with increased shoulder pain. Open procedures require more dynamic movements as compared with the relatively static nature of laparoscopy. ${ }^{10}$

A static uninterrupted posture will increase the risk of long-term back pain through a sustained isometric contraction of the lumbar extensor muscle group. ${ }^{26}$ Even if a force is generated at $2 \%$ of the muscle's maximum voluntary contraction, this results in decreased tissue oxygenation and risks subsequent injury. ${ }^{7}$ Further injury occurs as muscles and tendons accumulate lactic acid and toxins secondary to the compression of veins and capillaries when maintained in these static positions. ${ }^{38,39}$ This further exacerbates tissue trauma due to decreased oxygenation and lack of nutrition. ${ }^{40}$ During minimally invasive procedures, $70 \%$ of surgeons tend to maintain a static posture, as these operations require less back flexion compared with open procedures. These infrequent changes in position over time will result in muscular strain and fatigue. ${ }^{38,41,42}$ As procedural time increases, forward flexion of the torso intensifies. This position increases the compressive load on the spine, resulting in fatigue, stress, and pain. All factors associated with a reduction in performance. ${ }^{43}$

Improper head and neck angles result in cervical discomfort and have also been associated with posture fatigue. Numbness, stiffness, and pain in the shoulders, neck, arm, and wrists have been reported by 8 to $18 \%$ of surgeons. ${ }^{26}$ 
During open procedures, surgeons have a propensity to create a forward flexed neck angle of 60 to 90 degrees, and intraoperative electromyography (EMG) studies have demonstrated a greater amplitude in the cervical erector spinal muscles with this posture. This results in a greater workload on the cervical extensor muscle group to stabilize the head. ${ }^{10}$ In addition to neck and back pain, this anterior tilt of the neck is correlated with MSK symptoms in the trapezius and the temporomandibular joint. This posture is accentuated in those with preexisting symptoms, further worsening pain and discomfort over time. ${ }^{44}$

Standing upright places an intermediate amount of compression on the spinal column, which ultimately results in lower back pain. ${ }^{32}$ Due to the force of gravity, improper posture will accentuate cervical lordosis and thoracic kyphosis to maintain lumbar lordosis. This effect on the spinal column results in an altered biomechanical loading on muscles, ligaments, and joints that can trigger long-term injuries. This abnormal compensation in position may ultimately result in a decrease in truncal height. ${ }^{32}$ These hazards are directly related to the duration of exposure and underscore the importance of surgeons being mindful of their posture and performing corrective measures periodically while operating.

\section{Laparoscopy}

The advent of laparoscopy has been associated with considerable benefit in patient outcomes. This approach has been correlated with decreased postoperative pain, less wound complications, and a shorter length of hospital stay. ${ }^{45}$ However, minimally invasive surgery is technically demanding and imposes a significant physical demand on the surgeon. Park et al report that $87 \%$ of surgeons who regularly perform minimally invasive operations have succumbed to work-related symptoms and injuries directly related to this approach. ${ }^{21}$ The greatest stress on the body during laparoscopy has been reported to be on both the cervical and lumbar spine, in addition to the shoulders, wrists, and hands. MSK disorders in these body segments are secondary to both static positions and awkward postures. ${ }^{39,46,47}$ Furthermore, internal rotation movements of the shoulder, as well as supination, ulnar, and radial deviations occur more frequently in laparoscopic procedures compared with open procedures, which are associated with a greater rate of discomfort in these regions. ${ }^{38}$

Laparoscopy requires a substantial amount of focus, and the degree of concentration endured by these procedures is reportedly greater than that by open surgery. ${ }^{38}$ It is important that the surgeon understands the risks of operating laparoscopically, and although there are multiple barriers to ergonomic efficiency, success begins with surgeon awareness and recognition of existing obstacles. ${ }^{24}$ Ergonomic guidelines for laparoscopic equipment have also been developed and will benefit the surgeon when implemented ( - Table 1). ${ }^{48}$ Reports have demonstrated that as few as $9 \%$ of surgeons are even aware of any formalized recommendations, and only 3\% apply them routinely. ${ }^{49}$ One of the fundamental concerns with poor ergonomics in the operating room is associated with the
Table 1 Ergonomics principles and recommendations for laparoscopic procedures

\begin{tabular}{|c|}
\hline Ideal working posture \\
\hline Stand upright \\
\hline Avoid static positions \\
\hline Arm and elbow angle between 90 and 120 degrees \\
\hline Forearm in a neutral horizontal position \\
\hline Slight inward rotation of the arms \\
\hline Mild wrist extension and finger flexion \\
\hline Table height \\
\hline Table between 66 and $77 \mathrm{~cm}$ above the floor \\
\hline Purchase of tables that can be lowered adequately \\
\hline Adjust for a neutral posture \\
\hline Instruments and elbows aligned \\
\hline Image display screen position \\
\hline Request ceiling-mounted screens \\
\hline Unobstructed and in-direct view of the operator \\
\hline Distance between 0.9 and $3 \mathrm{~m}$ from the surgeon \\
\hline $\begin{array}{l}\text { Viewing angle to monitor: } 15 \text { - to } 45 \text {-degree downward } \\
\text { gaze }\end{array}$ \\
\hline Cautery and insufflation setting in the surgeon's view \\
\hline Separate monitor for assistant \\
\hline Instrument handle design and usage \\
\hline $\begin{array}{l}\text { Ring handles: avoid excess pressure on fingers while } \\
\text { activating }\end{array}$ \\
\hline Avoid excess ulnar or radial deviation \\
\hline $\begin{array}{l}\text { Locking mechanism prevents tissue slippage or excessive } \\
\text { force }\end{array}$ \\
\hline Locking mechanism should rotate on/off \\
\hline $\begin{array}{l}\text { Avoid friction between the instrument and the abdominal } \\
\text { wall }\end{array}$ \\
\hline Handle manipulation for the left and right hands \\
\hline $\begin{array}{l}\text { Avoid pressure on the thenar eminence or the palm } \\
\text { activating instruments }\end{array}$ \\
\hline Use rotational handles \\
\hline $\begin{array}{l}\text { Ideal instrument manipulation angle: } 45-60 \text { degrees in } \\
\text { reference to the patient }\end{array}$ \\
\hline Trocar placement \\
\hline Triangulate toward the target anatomy \\
\hline Target organ $1520 \mathrm{~cm}$ from the center trocar \\
\hline Remaining trocars arranged in $15-20 \mathrm{~cm}$ arc, $5-7 \mathrm{~cm}$ apart \\
\hline $\begin{array}{l}\text { Avoid placement that requires excess reach over the } \\
\text { patient }\end{array}$ \\
\hline
\end{tabular}

surgeon's nonneutral body position, and this is often driven by their equipment and a failure to be mindful of their posture. As surgeons progress through the early laparoscopic learning curve, they should be encouraged to maintain a comfortable and natural body posture to avoid the fatigue and pain that occur as a result of cumulative bad habits (- Fig. 1).22,50 
There are inherent differences between the ergonomics of open and laparoscopic equipment, which imposes varying constraints on the operator. The use of open surgical instruments provides multiple degrees of freedom, allowing for dynamic movements, with dissection performed in a threedimensional space in line with the target organ. In contrast, during laparoscopy, the surgeon is typically found in a static posture limited by a two-dimensional view, with the loss of physical contact with the surgical field. This disconnect between visual and motor sensations alters the efficiency of the procedure. The loss of tactile feedback alters depth perception and adds to the complexity of the operation.

The ability to move laparoscopic instruments is restricted to only four degrees of freedom: rotational, up/down, left/right, in/out. ${ }^{51,52}$ In addition, the force necessary to grasp laparoscopic instruments is almost six times greater than what is required in open surgery. Accordingly, the surgeon grips the instruments harder, which is a less efficient transfer of energy from the handle to the tip. Holding the instruments in this fashion increases pain and discomfort throughout the hand. ${ }^{15,22,26}$ When laparoscopic instruments are used, they are passed through trocars fixed at the abdominal wall, and this creates a paradoxical fulcrum effect, where the intracorporeal aspect of the instrument moves in the opposite direction of the surgeon's hand. Stationary trocar positions will prohibit the surgeon from adjusting their body posture around the instruments, resulting in awkward working angles. ${ }^{51}$ Harmful forces may be exerted on the surgeon's torso when contorting his/her body around a morbidly obese patient or one with a thick abdominal wall. In addition, if trocars are skived, or not placed in a perpendicular direction between the skin and peritoneal cavity, excessive force to be exerted on the instruments will be required and may result in upper extremity discomfort. Operating laparoscopically is not intuitive. This alteration in spatial recognition overtime can affect surgeon performance. This approach requires a focused and learned coordination of movements to maintain mental stamina and prevent harm to the surgeon (- Table 2). ${ }^{15,22}$

Instrument length has also been associated with an increased risk of shoulder discomfort. The maneuverability of these instruments becomes increasingly difficult when the ratio of the intracorporeal to extracorporeal length is $<1$. A greater degree of extracorporeal length increases the distance of instrument movement, which creates a wider elbow-toshoulder angle and is associated with muscle fatigue and joint

\section{Table 2 Factors contributing to mental fatigue}

\begin{tabular}{|l|}
\hline $\begin{array}{l}\text { Fulcrum effect of instruments causes visualization of } \\
\text { inverted movements }\end{array}$ \\
\hline Increased level of hand-eye coordination \\
\hline Two-dimensional images \\
\hline Loss of depth perception \\
\hline Effects of light reflection and shadows \\
\hline Lack of efficiency in movement around the operative field \\
\hline Duration of surgery \\
\hline
\end{tabular}

pain. ${ }^{53}$ Laparoscopy is also associated with an increased risk of uncomfortable wrist positions and may lead to increased pain, numbness, and fatigue in the hand. ${ }^{38}$ In addition, laparoscopic handles are generally not adjustable, and some are created with cumbersome locking mechanisms. Opening and closing many of these instruments require an excessive and disproportionate amount of force on the thumb and differs based on hand size. Instruments are rarely manufactured in varying sizes to fit the hand dimensions of the surgeon, and this may result in discomfort, nerve compression, and limited maneuverability. ${ }^{54,55}$

Proper surgeon location at the operating room table and trocar placement both affect posture. Trocars should be triangulated, with the target organ approximately 15 to $20 \mathrm{~cm}$ from the center port. Additional trocars are placed in a semicircular pattern, with each trocar approximately 5 to $7 \mathrm{~cm}$ apart. Correct placement facilitates instrument working angles between 60 and 90 degrees and avoids excessive extracorporeal instrument length and friction from the abdominal wall. $^{22,56}$ If trocar positioning is too far from the target organ, it may cause the surgeon to push on the abdominal wall to gain a closer distance, causing muscle strain. In general, a laparoscopic procedure is performed with the surgeon on the opposite side of the patient's pathology. Reaching over or across the patient for trocar access is indicative of incorrect port placement. Standing between the patient's legs may force the surgeon to lean over the patient, placing excess stress on the back. ${ }^{38}$ However, an evaluation of laparoscopic cholecystectomies identified that operating while standing between the patient's legs may be more ergonomically suitable, as standing on the patient's left side was associated with increased stress on the upper arms, neck, and trunk due to a high rate of upper arm flexion, abduction, shoulder elevation, neck flexion, and torso rotation. ${ }^{57}$ These findings serve as an example that one position does not fit all operations, and, more importantly, the surgeon must be aware and knowledgeable of beneficial ergonomic and positioning options.

Sensorial ergonomics requires optimizing visualization to allow for precision, accuracy, and dexterity to manipulate tissue. $^{22}$ Positioning of the displayed monitor will affect visual orientation, neck position, and stance throughout a procedure. ${ }^{24}$ When feasible, the surgeon should request for operating rooms that are designed for laparoscopy. This typically entails multiple ceiling mounted screens that allow for easy manipulation and height adjustment. The main laparoscopic console should be in direct view of the surgeon or assistant to prevent rotation or poor posture to visualize equipment settings. ${ }^{24,58}$ The monitor height should be in front of the operator and approximately 10 to 25 degrees below the horizontal plane of the eye with a downward gaze (-Fig. 1). This promotes a neutral position of the cervical muscles and lowers the degree of neck strain while providing an optimal height for visualization. ${ }^{59,60}$ When the monitor is too high, this requires the surgeon to compensate by placing an excess amount of extension in the cervical and lumbar spine. ${ }^{36}$ The recommended distance between the image and the surgeon should be between 0.9 and $3 \mathrm{~m}$, a space that allows for ideal accommodation of the ocular lens (-Fig. 1). 
When the screen is too close, it increases eye strain; however, this measurement may be slightly altered based on the size of the screen. ${ }^{15}$ The assistant should have a separate screen, as even camera holding is a risk factor for work-related injuries. $^{22,24,61}$ Spending dedicated time to adjust the visual display screen will develop an environment conducive to operating safely, minimizing surgeon fatigue, and reducing the risk of adverse outcomes. ${ }^{24}$

Table height is critical to maintaining a neutral posture, and this applies to both laparoscopic and open procedures. The table should be at the height of the surgeon's elbow. ${ }^{38}$ When standing comfortably, the angle of the arm and elbow should be between 90 and 120 degrees, with the forearm in a neutral and horizontal position at a point between supination and pronation ( $\mathbf{- F i g . 1}$ ). The wrists should be placed in a slightly extended position, with a slight bend of the fingers. ${ }^{26,50}$ The surgeon should also be mindful of the height of his/her assistance or cosurgeon, as the ergonomic safety of all involved staff is critical to the efficiency and outcome of the operation.

When the table is too high, the surgeon is forced to raise his/her arms and change the angle of forearm and wrist to compensate for grip on the instruments. This causes fatigue and pain in the wrist and shoulders due to excessive extension in these joints. This discomfort makes fine dissection and suture placement challenging. It is common to rely on outdated equipment with limited motion, particularly in table height. The purchase of updated operating room tables may be necessary, requiring a capital investment by the hospital.

In addition, a table adjusted too high often requires a surgeon to stand on a step stool. This confines the surgeon to a smaller working area. When this space is cluttered by cables or a foot pedal, this could offset surgeon balance and cause unintentional motion and subsequent injury to the patient. Furthermore, the surgeon may succumb to an injury if one should fall off the platform. ${ }^{50}$ If a foot pedal is used for electrocautery, this should be at the same level as the surgeon's foot and aligned with the working instrument toward the targeted anatomy (-Fig. 1). This will prevent any unnecessary shift in the legs or feet, or an awkward position during cauterization of tissue. For extra protection, consider always placing two platforms adjacent to each other, as well as two platforms behind, to prevent an inadvertently fall off the back side of the stool.

Patient positioning on the table requires careful consideration. When the arms are placed at 90 degrees to the bed, it may interfere with the surgeon's stance in relation to the target intra-abdominal quadrant. In these circumstances, the patient's arms should be tucked to allow the surgeon to take a comfortable stance at the operating table. Any position that results in the surgeon twisting his/her upper extremity, lower extremity, or back should be avoided. ${ }^{36}$ Developing a comfortable work environment by incorporating these ergonomic principles and recommendations will minimize physical fatigue, help avoid injury, and reduce the additive mental stress experienced during lengthy laparoscopic procedures.

\section{Robotic Surgery}

Offering an advantage over the ergonomic limitations of laparoscopy, robot-assisted surgery provides a different perspective of the operative field. The da Vinci robotic surgery system (Intuitive Surgical, Sunnyvale, CA) was introduced in 2003 and has become increasingly popular throughout multiple surgical disciplines. ${ }^{62}$ This system allows the operation to be conducted at a console away from the patient, with the surgeon in a seated position. The robotic arms require little mechanical effort to activate. The wristed instruments allow for seven degrees of freedom, which aids in fine manipulation. In addition, the camera display is three-dimensional and set up with a downward gaze, minimizing potential eyes strain and fatigue. The console itself can be adjusted to a personalized optimal ergonomic position, and a supportive chair can be employed as well.

Zihni et al performed the first quantitative analysis comparing ergonomic stress between laparoscopic and robotic procedures. The authors used surface EMG to compare the activation of the bicep, triceps, deltoid, and trapezius muscle groups during both laparoscopic and robotic procedures. Results demonstrated a significantly elevated level of muscle activation in all muscle groups, except for the trapezius muscle, during laparoscopy when compared with robotic surgery. ${ }^{63}$ The suggested etiology of increased trapezius muscle activation during robotic surgery may be secondary to the anterior tilt of the head and neck while looking down into the console. In a smaller pilot study, Lawson et al demonstrated postural advantages during robotic procedures. The authors identified decreased discomfort in the arm, forearm, and wrists; however, there was a greater rate of neck strain due to the position of the upper torso. ${ }^{47}$ Overall, these findings concluded that laparoscopy had a higher degree of ergonomic challenges based on a greater measurement of muscle activation compared with a robotic approach. ${ }^{63}$ In a recent study by Franasiak et al, $45 \%$ of surveyed surgeons reported experiencing strain while performing a robotic operation, with $>70 \%$ of these surgeons reporting neck discomfort. The authors identified that only $16 \%$ of surgeons underwent any formal ergonomic training; however, when an educational program was implemented, $74 \%$ noted a decrease in strain when implementing the recommended positions. ${ }^{20}$ Although the robotic surgical platform addresses and alleviates many of the ergonomic deficiencies associated with conventional laparoscopy, this modality has not fully alleviated the risk of MSK discomfort among surgeons. ${ }^{64}$

During robotic procedures, the surgeon should always focus on a neutral position to avoid static loading on the body. ${ }^{65}$ Once this is established, the surgeon must continuously reevaluate themselves to ensure that no pain or discomfort develops. At the console, the surgeon's chair should be on lockable wheels and have adjustable height and depth, as well as lumbar support. The feet should rest comfortably on the ground in front of the pedals, with the knees at a 90-degree angle or greater. The arms should be perpendicular to the floor, and the elbows should be at 
90 degrees resting comfortably on the armrest while keeping the elbows tucked in closely to off-load pressure and reduce discomfort along the torso. Frequent clutching of the mechanical arms is imperative to maintain the elbows in this position. The optical camera display should be placed at a comfortable height for viewing. The ocular height should be adjusted to prevent or relieve neck, shoulder, and upper back strain. There should be no arching or straightening of the back or neck to increase height. The neck should be at a downward angle of approximately 20 degrees, without any excess bending. When positioned, the surgeon should avoid placing unnecessary tension or force on the headrest, as this can increase forehead pain and neck discomfort. No longer constrained by a sterile field, if strain or discomfort is recognized, the surgeon can stand, flex, and/or stretch for small periods of time. This can be performed routinely during small breaks for instrument or suture exchanges. The chair and console height can be readjusted at any time to provide a more comfortable position. ${ }^{20,65}$ Due to the paucity of research comparing the ergonomics of robotassisted surgery versus conventional laparoscopy, further investigations are required to fully elucidate the surgeon's risk of work-related injury when using this technology. ${ }^{66}$

\section{Musculoskeletal Therapy}

Surgeons who suffer from MSK injuries employ multiple options to address their symptoms, including nonsteroid anti-inflammatory medications, muscle relaxants, and additional treatments such as massage, acupuncture, and physical therapy. ${ }^{67}$ The risk of narcotic use and dependence should not be ignored. ${ }^{68}$ Surgeons who suffer from a work-related injury may require an increased amount of sick leave, variations or limitations to their practice, temporary assignments to nonsurgical tasks, or early retirement. $^{4,19,69}$ Self-medication or analgesic usage may alleviate symptoms, but these treatment options do not target the underlying cause. The ultimate goal would be to recognize causative factors at early stages and employ countermeasures to minimize pain and disability while promoting career longevity.

Spinal instability is one of the most common etiologies of lower back pain and one of the primary disorders affecting surgeons. Improving stability decreases the mechanical irritation of the spinal column and reduces symptoms. ${ }^{70,71}$ Surgeons may require orthopedic footwear, spinal braces, and exoskeletons to maintain stability and posture while operating. ${ }^{69}$ Devices including ergonomic body supports and headrests have been piloted to assist in creating a more stable and natural posture to alleviate strain on the cervical, thoracic, and lumbar spine during both open and laparoscopic procedures. ${ }^{72,73}$ The implementation of standing on a Gel Mat (Gelpro, Austin, TX) has been evaluated, and surgeons reported an improved operative posture and less back, knee, and foot pain with this intervention. The overall subjective perception of physical discomfort and fatigue was significantly less, and surgeons also reported a lower rate of intraoperative errors when standing on the Gel Mat. ${ }^{74}$
The ergonomics of surgery need not solely rely on equipment, as there are several measures a surgeon can employ in the operating room that will protect his/her MSK health. For example, alternating surgeon participation during complex and lengthy operations allows for periodic rest breaks. Cuschieri recommended routine 10 -minute breaks every 2 hours where the operating team and surgeon leave the room. ${ }^{52}$ While this might appear extreme, the concept and benefits of microbreaks have been well established. A multicenter study performed by Hallbeck et al implemented the use of 1- to 2-minute exercise-guided breaks spaced every 20 to 40 minutes of operative time or when medically appropriate. These activities focused on rotational movements and activation of muscle groups in the neck, back, hands, and lower extremities during procedures lasting between 2 and 12 hours. Results demonstrated that microbreaks did not increase operative duration, disrupt the workflow of the procedure, or cause any distractions. Surgeons reported a $34 \%$ improvement in mental focus and a 57\% improvement in physical performance, and following this investigation, almost $90 \%$ of surgeons desired to incorporate microbreaks into their practice. ${ }^{75}$ Park et al further investigated the effects of targeted stretching microbreaks using similar time intervals. These exercises preserved sterility and involved neck flexion, extension, and lateral rotation; backward shoulder roll and chest stretch; upper back and hand stretch; low back flexion and extension; gluteus maximus contractions; and foot and heel lifts for lower extremity and ankle stretches. Encouraging results were demonstrated with an improvement in both physical and mental performance, with no impact on case duration. Furthermore, the majority of surgeons planned to continue this routine regularly. ${ }^{8}$ These two studies clearly demonstrate a safe, effective, and efficient method to reduce MSK discomfort during the conduct of surgery. 8,75

Simple postural rests, even if these are not formal procedure breaks, should be implemented to readjust the body to a neutral stance and alleviate tension on the torso and extremities. ${ }^{61}$ Additional sterile intraoperative stretches and exercises may be performed to offset strain on the body. The upper extremity can be mobilized with bilateral or unilateral arm raises. This allows for full shoulder elevation, external rotation, scapular depression, and retraction to reduce strain on this location. The scapula is drawn down and the back while the sternum is lifted slightly to allow the shoulder girdle to be stretched and mobilized. Squatting is another movement that offsets pressure on the torso. This multifunctional exercise engages the large muscles of the lower extremity and recapitulates symmetrical weightbearing and a neutral spine. This can be performed without compromising the sterility of the operation. ${ }^{36}$

Furthermore, it is important to avoid any excessive or sustained shoulder abduction and internal rotation. This position is suboptimal as it requires the greatest workload on the deltoid and trapezius muscles, predisposing surgeons to fatigue and injury. The surgeon should remember to drop the shoulders and relax the hands, as this will off load the pressure on these muscle groups. To avoid unnecessary shear 
force on the pelvis and lower spine, one should refrain from any positions that shift weight onto one leg. This typically occurs when using a foot pedal at varying elevations to the ground, causing an asymmetrical load on the lower extremity. To further protect the torso and lower extremities, place the feet shoulder width apart, do not lock the knees, and abstain from shifting weight in the hips. If a circumstance arises that requires weight to be shifted, it should be countered by engaging the deep muscles of the trunk and pelvis to maintain a neutral position. Consideration of the operating room setup, maintaining a stable posture while standing or sitting, and repeatedly employing a practice of postural adjustments are critical to maintaining a long, healthy, and injury-free career in surgery. ${ }^{36}$

\section{Prevention through Conditioning}

Physical fitness is often correlated with strength and physiological variables such as heart rate, blood pressure, and oxygen consumption. However, joint flexibility and range of motion are often discordant with physical endurance, and when not conditioned properly, this results in injury. ${ }^{76,77}$ High-intensity functional training (HIT) programs have been rapidly expanding throughout all communities due to the benefits seen in body composition and strength. Even though advantages in health and wellness may be gained through these activities, it is imperative that novice athletes enroll in a well-organized and monitored introductory program to avoid injury and time away from work. ${ }^{78}$

Although multiple modalities exist that aid in reducing symptoms of lower back pain and MSK discomfort, establishing a program of injury prevention is of even greater importance. The spine is naturally unstable, and it is the interplay of multiple muscles groups that allows for coordinated contractions to sustain a functional position. ${ }^{70}$ Pain from mechanical stress, tension, lack of joint mobility, an imbalance of muscular length, strength, and endurance, lack of stabilization, and decreased cardiopulmonary stamina will all impact posture. Interventions often addressed through physical therapy involve techniques to foster spinal and extremity movements, limb stretching, and joint mobilization, as well as stabilization exercises with progression to strength, functional, and aerobic training. Furthermore, muscle groups from the suboccipital region down to the gastrocnemius are interrelated in the development of a strong, stable spine. Functional, stretching, and strengthening exercises can all be applied to each specific body region that requires conditioning and should be tailored to the individual surgeon in relation to his/her work environment and demands. ${ }^{34}$

There are various programs, resources, and routines that can be implemented to manage MSK injuries as well as prevent them in the healthy surgeon. These should be customized, as the mechanical etiology of instability and pain will vary among individuals. Although the breadth of techniques to prevent and improve MSK disorders cannot all be discussed here, it is important that before adopting any functional movement or strengthening program, one should receive appropriate education from an accredited therapist. Improving the strength and stability of the lumbopelvic hip complex or core provides stabilization to the torso and spine. This requires balance, plyometric, and strengthening routines to focus on a neutral spine with optimal trunk positioning to transfer weight-bearing loads without harm. Any program should be performed in a stepwise fashion beginning with local muscle recruitment, then stabilization postures, and, finally, dynamic body exercises. ${ }^{79}$ Training of the deep trunk muscles has been associated with a reduction in chronic lower back pain. However, McGill and Karpowicz recommend the use of a stability training program that places minimal force on the spine, as many people who require these routines have compromised stability at baseline. ${ }^{80,81}$

Suni et al evaluated the effectiveness of conditioning and strengthening the normal lordotic curve of the spine, referred to as the "neutral zone." Targeting this region facilitates the prevention of lower back pain by decreasing the effects of harmful loads placed on the lower back and subsequent ligamentous injuries. The studied exercises focused on the lumbar neutral zone and included balance, abdominal curl-up maneuvers, hip joint and hip flexor stretches, squat exercises, trunk muscle balance exercises on the hands and knees, upper body rotation with a rubber band, and upper body rotation while side-lying. These movements were initially guided by a physical therapist; however, patients had to demonstrate a commitment to the program, with graduated increments in activity, and expectations that these drills would be incorporated into the patients' daily schedule. The authors demonstrated a 39\% reduction in lower back pain in the treatment arm at 12 months. Additionally, at the conclusion of the study, those in the treatment group had an improved outlook on their future ability to work. ${ }^{82}$

In addition to standard strengthening activities, maximizing the effects of intra-abdominal pressure through the coordination of trunk and respiratory muscles directly affects pelvic stability and torso stabilization. Exhalation workouts target an increase in intra-abdominal pressure, resulting in the activation of abdominal and trunk muscles. During breathing exercises, the contraction of the abdominal wall is controlled and spinal stabilization can be accomplished ${ }^{83,84}$ This method of breathing allows for the relaxation of lumbar muscles and an improvement in pelvic mobility, and may effectively relieve and prevent back pain. ${ }^{85}$ When trained properly, the surgeon should feel empowered to practice all these exercises routinely to improve mobility, enhance flexibility, and, ultimately, decrease pain.

Besides muscle-directed exercises, additional activities have demonstrated benefit in core strength and posture improvement. Yoga has been reported to promote both physical and psychological health. ${ }^{86,87}$ Isha hot yoga is a practice of unique poses performed in a warm environment. This particular subset of yoga has been correlated with an improvement in spinal flexibility and overall functional capacity. ${ }^{88,89}$ In a review of more than 100 patients participating in a hot yoga training schedule, Kumar et al 
demonstrated a significant progression in core stability and single-leg balance. This was analyzed through assessing a patient's ability to stand on one leg while keeping the opposite foot on the inside of their knee for support, as well as a plank test that involves placement in the prone position, with a rise up on the toes, and bending the forearms while keeping the entire body stiff as a plank. This particular routine has been validated to assess core stability. This form of hot yoga appears to develop muscle activity and improve the muscular endurance responsible for controlling these dynamic postures. ${ }^{90}$

Pilates has also been used in the treatment and prevention of lower back pain. This method of conditioning focuses on improving posture through lumbopelvic stability exercises and breath control. The activation of the body's core muscles, the diaphragm, and pelvic floor muscles will develop the mobility and flexibility associated with spinal support and may reduce lower back and hip pain. ${ }^{91}$ Furthermore, Pilates teaches the correct timing of muscle activation, another factor that, when not optimized, contributes to pain syndromes. Valenza et al randomized participants to an 8 -week trial of Pilates where this intervention group attended class twice a week for 45 minutes, performing 14 various floor exercises at basic, intermediate, and advanced levels. Participants evaluated after Pilates had a significant reduction in chronic pain and disabilities scores, in addition to an improvement in flexibility and balance. ${ }^{92}$

Osteopathic manipulation and targeted manual therapies have also been implemented with the goal of alleviating the underlying etiology of pain. This area of medicine focuses on the entire body as a system and provides a hands-on technique to alleviate discomfort, restore function, and promote health. Cruser et al evaluated the effects of manipulative therapy on a military population with acute lower back pain. Interventions were performed weekly over 1 month and included a mixture of high- and moderate-velocity thrusts, soft tissue stretching, kneading and pressure, myofascial release and stretching, positional treatments of myofascial tender points, and muscle energy techniques. These patients were found to have a greater reduction in pain, with a higher rate of self-improvement and treatment satisfaction. ${ }^{93}$ Myofascial release has also been directed to treat lower back pain among a cohort of nurses. This technique uses the application of a low-load long-duration stretch to the muscle's fascia with the purpose of restoring length to improve function and reduce pain. This safe and effective technique can be administered by most therapists. Nurses were found to have a 53\% reduction in pain and a $30 \%$ reduction in disability following 8 weeks of therapy. ${ }^{94}$ These treatment methods, when received as an adjunct to a regular exercise program for spinal strength and stability, may significantly improve longterm health and wellness.

\section{Conclusion}

Across all surgical approaches and platforms, the conduct of an operation may put the surgeon at risk for developing chronic MSK injuries. In such a physically demanding profes- sion, injury prevention is critical. This can be achieved through a combination of optimizing operating room ergonomics and health maintenance through tailored training regimens. Most surgeons lack awareness and proper training related to operative posture, ergonomics, and risk mitigation through practice changes, modifications of the working environment, and prevention strategies. The risk to MSK health is cumulative and rarely recognized until injury occurs, impacting productivity, job satisfaction, and, ultimately, career longevity. Through awareness and advocacy, we can enhance surgeon health and maintain a productive workforce that is prepared and conditioned to provide surgical care for our patients.

\section{Disclosure}

This is an original work by the authors. The opinions expressed are the authors' and authors' alone. They do not necessarily reflect the opinion of the United States Government, U.S. Department of Defense, or Madigan Army Medical Center.

\section{Conflict of Interest}

None.

\section{References}

1 Oakes K. 'This has to change': Surgeon pain, disability on the rise. ACS News Surgery, February 5, 2018

2 Patti MG, Schlottmann F, Sarr MG. The problem of burnout among surgeons. JAMA Surg 2018;153(05):403-404

3 Capone AC, Parikh PM, Gatti ME, Davidson BJ, Davison SP. Occupational injury in plastic surgeons. Plast Reconstr Surg 2010;125 (05):1555-1561

4 Soueid A, Oudit D, Thiagarajah S, Laitung G. The pain of surgery: pain experienced by surgeons while operating. Int J Surg 2010;8 (02):118-120

5 Sivak-Callcott JA, Diaz SR, Ducatman AM, Rosen CL, Nimbarte AD, Sedgeman JA. A survey study of occupational pain and injury in ophthalmic plastic surgeons. Ophthal Plast Reconstr Surg 2011; 27(01):28-32

6 Buckle PW, Devereux JJ. The nature of work-related neck and upper limb musculoskeletal disorders. Appl Ergon 2002;33(03): 207-217

7 McGill SM, Hughson RL, Parks K. Lumbar erector spinae oxygenation during prolonged contractions: implications for prolonged work. Ergonomics 2000;43(04):486-493

8 Park AE, Zahiri HR, Hallbeck MS, et al. Intraoperative "micro breaks" with targeted stretching enhance surgeon physical function and mental focus: a multicenter cohort study. Ann Surg 2017; 265(02):340-346

9 Wauben LS, van Veelen MA, Gossot D, Goossens RH. Application of ergonomic guidelines during minimally invasive surgery: a questionnaire survey of 284 surgeons. Surg Endosc 2006;20(08):1268-1274

10 Szeto GP, Ho P, Ting AC, Poon JT, Tsang RC, Cheng SW. A study of surgeons' postural muscle activity during open, laparoscopic, and endovascular surgery. Surg Endosc 2010;24(07):1712-1721

11 Berguer R. Surgery and ergonomics. Arch Surg 1999;134(09): 1011-1016

12 Berguer R, Hreljac A. The relationship between hand size and difficulty using surgical instruments: a survey of 726 laparoscopic surgeons. Surg Endosc 2004;18(03):508-512

13 Lawther RE, Kirk GR, Regan MC. Laparoscopic procedures are associated with a significant risk of digital nerve injury for general surgeons. Ann R Coll Surg Engl 2002;84(06):443 
14 Blackwell JR, Kornatz KW, Heath EM. Effect of grip span on maximal grip force and fatigue of flexor digitorum superficialis. Appl Ergon 1999;30(05):401-405

15 Reyes DA, Tang B, Cuschieri A. Minimal access surgery (MAS)-related surgeon morbidity syndromes. Surg Endosc 2006;20(01):1-13

16 Davis WT, Fletcher SA, Guillamondegui OD. Musculoskeletal occupational injury among surgeons: effects for patients, providers, and institutions. J Surg Res 2014;189(02):207-212.e6

17 Waehrer G, Leigh JP, Miller TR. Costs of occupational injury and illness within the health services sector. Int J Health Serv 2005;35 (02):343-359

18 Leigh JP. Economic burden of occupational injury and illness in the United States. Milbank Q 2011;89(04):728-772

19 Epstein S, Sparer EH, Tran BN, et al. Prevalence of work-related musculoskeletal disorders among surgeons and interventionalists: a systematic review and meta-analysis. JAMA Surg 2018;153 (02):e174947

20 Franasiak J, Craven R, Mosaly P, Gehrig PA. Feasibility and acceptance of a robotic surgery ergonomic training program. JSLS 2014; 18(04):00166

21 Park A, Lee G, Seagull FJ, Meenaghan N, Dexter D. Patients benefit while surgeons suffer: an impending epidemic. J Am Coll Surg 2010;210(03):306-313

22 Supe AN, Kulkarni GV, Supe PA. Ergonomics in laparoscopic surgery. J Minim Access Surg 2010;6(02):31-36

23 Stone R, McCloy R. Ergonomics in medicine and surgery. BMJ 2004;328(7448):1115-1118

24 Horst N, Krokowicz P. Risk for the operator related to laparoscopic surgery and selected measures of their minimization. Pol Przegl Chir 2011;83(06):347-351

25 Seagull FJ. Disparities between industrial and surgical ergonomics. Work 2012;41(01, Suppl 1):4669-4672

26 Berguer R, Forkey DL, Smith WD. Ergonomic problems associated with laparoscopic surgery. Surg Endosc 1999;13(05):466-468

27 Gilbreth FB. Motion study in surgery. Can J Med Surg 1916;40:22-31

28 Taylor F. The Principles of Scientific Management. New York, NY: Harper and Bros; 1929

29 Bragg TL. An ergonomics program for the health care setting. Nurs Manage 1996;27(07):58-61, quiz 62

30 Rundcrantz BL, Johnsson B, Moritz U. Occupational cervico-brachial disorders among dentists. Analysis of ergonomics and locomotor functions. Swed Dent J 1991;15(03):105-115

31 Harris NO, Crabb LJ. Ergonomics. Reducing mental and physical fatigue in the dental operatory. Dent Clin North Am 1978;22(03): 331-345

32 Vieira ER, Kumar S. Working postures: a literature review. J Occup Rehabil 2004;14(02):143-159

33 Kim D, Cho M, Park Y, Yang Y. Effect of an exercise program for posture correction on musculoskeletal pain. J Phys Ther Sci 2015; 27(06):1791-1794

34 Kisner C, Colby LA. The spine and posture: structure, function, postural impairments, and management guidelines. In: Therapeutic Exercise: Foundations and Techniques. Philadelphia, PA: F. A. Davis Company; 2007:383-406

35 Kumar S. Theories of musculoskeletal injury causation. Ergonomics 2001;44(01):17-47

36 Rosenblatt PL, McKinney J, Adams SR. Ergonomics in the operating room: protecting the surgeon. J Minim Invasive Gynecol 2013; 20(06):744

37 Szeto GP, Cheng SW, Poon JT, Ting AC, Tsang RC, Ho P. Surgeons' static posture and movement repetitions in open and laparoscopic surgery. J Surg Res 2012;172(01):e19-e31

38 Nguyen NT, Ho HS, Smith WD, et al. An ergonomic evaluation of surgeons' axial skeletal and upper extremity movements during laparoscopic and open surgery. Am J Surg 2001;182(06):720-724

39 Berguer R, Rab GT, Abu-Ghaida H, Alarcon A, Chung J. A comparison of surgeons' posture during laparoscopic and open surgical procedures. Surg Endosc 1997;11(02):139-142
40 Kumar S. A conceptual model of overexertion [correction of overexertion], safety, and risk of injury in occupational settings. Hum Factors 1994;36(02):197-209

41 Radermacher K, von Pichler KC, Erbse S, et al. Using human factor analysis and VR simulation techniques for the optimization of the surgical worksystem. Stud Health Technol Inform 1996; 29:532-541

42 Kant IJ, de Jong LC, van Rijssen-Moll M, Borm PJ. A survey of static and dynamic work postures of operating room staff. Int Arch Occup Environ Health 1992;63(06):423-428

43 Bhatnager V, Drury CG, Schiro SG. Posture, postural discomfort, and performance. Hum Factors 1985;27(02):189-199

44 Szeto GP, Straker L, Raine S. A field comparison of neck and shoulder postures in symptomatic and asymptomatic office workers. Appl Ergon 2002;33(01):75-84

45 Keller DS, Delaney CP, Hashemi L, Haas EM. A national evaluation of clinical and economic outcomes in open versus laparoscopic colorectal surgery. Surg Endosc 2016;30(10):4220-4228

46 Vereczkel A, Bubb H, Feussner H. Laparoscopic surgery and ergonomics: it's time to think of ourselves as well. Surg Endosc 2003;17(10):1680-1682

47 Lawson EH, Curet MJ, Sanchez BR, Schuster R, Berguer R. Postural ergonomics during robotic and laparoscopic gastric bypass surgery: a pilot project. J Robot Surg 2007;1(01):61-67

48 van Veelen MA, Nederlof EAL, Goossens RHM, Schot CJ, Jakimowicz JJ. Ergonomic problems encountered by the medical team related to products used for minimally invasive surgery. Surg Endosc 2003;17(07):1077-1081

49 Toffola ED, Rodigari A, Di Natali G, Ferrari S, Mazzacane B. Posture and fatigue among surgeons in the operating room [in Italian]. G Ital Med Lav Ergon 2009;31(04):414-418

50 Matern U. Ergonomic deficiencies in the operating room: examples from minimally invasive surgery. Work 2009;33(02):165-168

51 Patkin M, Isabel L. Ergonomics, engineering and surgery of endosurgical dissection. J R Coll Surg Edinb 1995;40(02):120-132

52 Cuschieri A. Whither minimal access surgery: tribulations and expectations. Am J Surg 1995;169(01):9-19

53 Emam TA, Hanna GB, Kimber C, Dunkley P, Cuschieri A. Effect of intracorporeal-extracorporeal instrument length ratio on endoscopic task performance and surgeon movements. Arch Surg 2000;135(01): 62-65, discussion 66

54 Kano N, Yamakawa T, Ishikawa Y, Miyajima N, Ohtaki S, Kasugai H. Prevention of laparoscopic surgeon's thumb. Surg Endosc 1995;9 (06):738-739

55 Lee WJ, Chae YS. Superficial nerve damage of thumb of laparoscopic surgeon. Surg Laparosc Endosc Percutan Tech 2001;11(03): 207-208

56 Trejo A, Jung MC, Oleynikov D, Hallbeck MS. Effect of handle design and target location on insertion and aim with a laparoscopic surgical tool. Appl Ergon 2007;38(06):745-753

57 Youssef Y, Lee G, Godinez C, et al. Laparoscopic cholecystectomy poses physical injury risk to surgeons: analysis of hand technique and standing position. Surg Endosc 2011;25(07):2168-2174

58 Kelts GI, McMains KC, Chen PG, Weitzel EK. Monitor height ergonomics: a comparison of operating room video display terminals. Allergy Rhinol (Providence) 2015;6(01):28-32

59 Menozzi M, von Buol A, Krueger $\mathrm{H}$, Miège $\mathrm{C}$. Direction of gaze and comfort: discovering the relation for the ergonomic optimization of visual tasks. Ophthalmic Physiol Opt 1994;14(04):393-399

60 Hanna GB, Shimi SM, Cuschieri A. Task performance in endoscopic surgery is influenced by location of the image display. Ann Surg 1998;227(04):481-484

61 Lee G, Lee T, Dexter D, et al. Ergonomic risk associated with assisting in minimally invasive surgery. Surg Endosc 2009;23(01): 182-188

62 Damle A, Damle RN, Flahive JM, et al. Diffusion of technology: trends in robotic-assisted colorectal surgery. Am J Surg 2017;214 (05):820-824 
63 Zihni AM, Ohu I, Cavallo JA, Cho S, Awad MM. Ergonomic analysis of robot-assisted and traditional laparoscopic procedures. Surg Endosc 2014;28(12):3379-3384

64 Yu D, Dural C, Morrow MM, et al. Intraoperative workload in robotic surgery assessed by wearable motion tracking sensors and questionnaires. Surg Endosc 2017;31(02):877-886

65 Lux MM, Marshall M, Erturk E, Joseph JV. Ergonomic evaluation and guidelines for use of the daVinci Robot system. J Endourol 2010;24(03):371-375

66 Dalager T, Søgaard K, Bech KT, Mogensen O, Jensen PT. Musculoskeletal pain among surgeons performing minimally invasive surgery: a systematic review. Surg Endosc 2017;31(02):516-526

67 Weiss J, Quante S, Xue F, Muche R, Reuss-Borst M. Effectiveness and acceptance of acupuncture in patients with chronic low back pain: results of a prospective, randomized, controlled trial. J Altern Complement Med 2013;19(12):935-941

68 McLellan AT, Skipper GS, Campbell M, DuPont RL. Five year outcomes in a cohort study of physicians treated for substance use disorders in the United States. BMJ 2008;337:a2038

69 Janki S, Mulder EEAP, IJzermans JNM, Tran TCK. Ergonomics in the operating room. Surg Endosc 2017;31(06):2457-2466

70 McGill SM, Grenier S, Kavcic N, Cholewicki J. Coordination of muscle activity to assure stability of the lumbar spine. J Electromyogr Kinesiol 2003;13(04):353-359

71 Ha SM, Oh JS, Jeon IC, Kwon OY. The effects of surface condition on abdominal muscle activity during single-legged hold exercise. J Electromyogr Kinesiol 2015;25(01):28-33

72 Albayrak A, van Veelen MA, Prins JF, Snijders CJ, de Ridder $\mathrm{H}$, Kazemier G. A newly designed ergonomic body support for surgeons. Surg Endosc 2007;21(10):1835-1840

73 Mitre AI, Duarte RJ, Arap MA, et al. Ergonomic aspects related to surgeon position in laparoscopic radical prostatectomy. J Endourol 2009;23(02):259-262

74 Haramis G, Rosales JC, Palacios JM, et al. Prospective randomized evaluation of FOOT gel pads for operating room staff COMFORT during laparoscopic renal surgery. Urology 2010;76(06):1405-1408

75 Hallbeck MS, Lowndes BR, Bingener J, et al. The impact of intraoperative microbreaks with exercises on surgeons: a multi-center cohort study. Appl Ergon 2017;60:334-341

76 Frost D, Andersen J, Lam T, Finlay T, Darby K, McGill S. The relationship between general measures of fitness, passive range of motion and whole-body movement quality. Ergonomics 2013; 56(04):637-649

77 Knapik JJ, Sharp MA, Canham-Chervak M, Hauret K, Patton JF, Jones BH. Risk factors for training-related injuries among men and women in basic combat training. Med Sci Sports Exerc 2001;33 (06):946-954

78 Claudino JG, Gabbett TJ, Bourgeois F, et al. CrossFit overview: systematic review and meta-analysis. Sports Med Open 2018;4 (01): 11

79 Huxel Bliven KC, Anderson BE. Core stability training for injury prevention. Sports Health 2013;5(06):514-522
80 Chang WD, Lin HY, Lai PT. Core strength training for patients with chronic low back pain. J Phys Ther Sci 2015;27(03):619-622

81 McGill SM, Karpowicz A. Exercises for spine stabilization: motion/ motor patterns, stability progressions, and clinical technique. Arch Phys Med Rehabil 2009;90(01):118-126

82 Suni J, Rinne M, Natri A, Statistisian MP, Parkkari J, Alaranta H. Control of the lumbar neutral zone decreases low back pain and improves self-evaluated work ability: a 12-month randomized controlled study. Spine 2006;31(18):E611-E620

83 Pulkovski N, Mannion AF, Caporaso F, et al. Ultrasound assessment of transversus abdominis muscle contraction ratio during abdominal hollowing: a useful tool to distinguish between patients with chronic low back pain and healthy controls? Eur Spine J 2012;21(Suppl 6):S750-S759

84 Talasz H, Kremser C, Kofler M, Kalchschmid E, Lechleitner M, Rudisch A. Phase-locked parallel movement of diaphragm and pelvic floor during breathing and coughing-a dynamic MRI investigation in healthy females. Int Urogynecol J Pelvic Floor Dysfunct 2011;22(01):61-68

85 Kang JI, Jeong DK, Choi H. Effect of exhalation exercise on trunk muscle activity And Oswestry Disability Index of patients with chronic low back pain. J Phys Ther Sci 2016;28(06):1738-1742

86 Ross A, Thomas S. The health benefits of yoga and exercise: a review of comparison studies. J Altern Complement Med 2010;16 (01):3-12

87 Varambally S, Gangadhar BN. Yoga: a spiritual practice with therapeutic value in psychiatry. Asian J Psychiatr 2012;5(02): 186-189

88 Haldavnekar RV, Tekur P, Nagarathna R, Nagendra HR. Effect of yogic colon cleansing (Laghu Sankhaprakshalana Kriya) on pain, spinal flexibility, disability and state anxiety in chronic low back pain. Int J Yoga 2014;7(02):111-119

89 Akhtar P, Yardi S, Akhtar M. Effects of yoga on functional capacity and well being. Int J Yoga 2013;6(01):76-79

90 Kumar S, Prasad S, Balakrishnan B, Muthukumaraswamy K, Ganesan M. Effects of Isha hatha yoga on core stability and standing balance. Adv Mind Body Med 2016;30(02):4-10

91 Battié MC, Bigos SJ, Fisher LD, et al. Anthropometric and clinical measures as predictors of back pain complaints in industry: a prospective study. J Spinal Disord 1990;3(03):195-204

92 Valenza MC, Rodríguez-Torres J, Cabrera-Martos I, Díaz-Pelegrina A, Aguilar-Ferrándiz ME, Castellote-Caballero Y. Results of a Pilates exercise program in patients with chronic non-specific low back pain: a randomized controlled trial. Clin Rehabil 2017; 31(06):753-760

93 Cruser A, Maurer D, Hensel K, Brown SK, White K, Stoll ST. A randomized, controlled trial of osteopathic manipulative treatment for acute low back pain in active duty military personnel. J Manual Manip Ther 2012;20:5-15

94 Ajimsha MS, Daniel B, Chithra S. Effectiveness of myofascial release in the management of chronic low back pain in nursing professionals. J Bodyw Mov Ther 2014;18(02):273-281 